\title{
Linker-Mediated Phase Behavior of DNA-Coated Colloids
}

\author{
Janna Lowensohn $\odot,{ }^{1}$ Bernardo Oyarzún, ${ }^{2}$ Guillermo Narváez Paliza, ${ }^{1}$ Bortolo M. Mognetti® ${ }^{2}$ and W. Benjamin Rogers $\oplus^{1, *}$ \\ ${ }^{1}$ Martin A. Fisher School of Physics, Brandeis University, Waltham, Massachusetts 02453, USA \\ ${ }^{2}$ Université Libre de Bruxelles, Interdisciplinary Center for Nonlinear Phenomena and Complex Systems, \\ Campus Plaine, Code Postal 231, Boulevard du Triomphe, B-1050 Brussels, Belgium
}

(Received 14 March 2019; revised manuscript received 13 August 2019; published 13 December 2019)

\begin{abstract}
The possibility of prescribing local interactions between nano- and microscopic components that direct them to assemble in a predictable fashion is a central goal of nanotechnology research. In this article, we advance a new paradigm in which the self-assembly of DNA-functionalized colloidal particles is programmed using linker oligonucleotides dispersed in solution. We find a phase diagram that is surprisingly rich compared to phase diagrams typical of other DNA-functionalized colloidal particles that interact by direct hybridization, including a reentrant melting transition upon increasing linker concentration, and show that multiple linker species can be combined to prescribe many interactions simultaneously. A new theory predicts the observed phase behavior quantitatively without any fitting parameters. Taken together, these experiments and model lay the groundwork for future research in programmable self-assembly, enabling the possibility of programming the hundreds of specific interactions needed to assemble fully addressable, mesoscopic structures, while also expanding our fundamental understanding of the unique phase behavior possible in colloidal suspensions.
\end{abstract}

DOI: 10.1103/PhysRevX.9.041054

Subject Areas: Soft Matter

\section{INTRODUCTION}

DNA-coated colloids are one of the most promising systems for designing complex self-assembling materials [1-3]. As in nature, the information required to specify the interactions and assembly pathways leading to a desired structure can be stored in the building blocks themselves. In the case of DNA-coated particles, this information is stored in the base sequences. In recent years, considerable progress has been made in using DNA to program the selfassembly of a variety of crystalline materials [4-8]. However, experimental demonstrations of the addressable assembly of DNA-coated particles into fully prescribed structures have yet to be realized.

Recent theoretical work highlights the challenges of using DNA-coated particles for assembling prescribed materials, which need not be symmetric or periodic $[9,10]$. To produce an arbitrary, complex structure with high yield from particles with specific, yet isotropic interactions, every particle must be different and have interactions chosen to favor the desired local configuration of the target structure [10]. Furthermore, all favorable

\footnotetext{
*wrogers@brandeis.edu
}

Published by the American Physical Society under the terms of the Creative Commons Attribution 4.0 International license. Further distribution of this work must maintain attribution to the author(s) and the published article's title, journal citation, and DOI. interactions must have comparable energies [9]. As a result, programming the assembly of even modest structures, which might contain only dozens of particles, requires specifying hundreds of unique binding interactions, all of which must have the same affinity.

In principle, DNA can encode these hundreds of interactions through careful design of the base sequences $[10,11]$. In practice, however, this potential is nearly impossible to realize in systems of DNA-coated particles interacting through direct binding of their grafted strands: The steep temperature dependence of the interactions [12-14], the inherent uncertainty in predictions of the binding affinities [15], and the inability to tune the relative interactions without resynthesizing the particles [16] make matching hundreds of unique interactions intractable. While strategies have been explored to reduce the number of specific interactions, including adding directional binding and exploiting hierarchical pathways to assembly $[17,18]$, these come with their own practical challenges.

An alternative approach is to design particles that interact through single-stranded DNA oligomers dissolved in solution instead of through direct binding of grafted strands. Here, the binding kinetics, the interaction strengths, and even the interaction matrix itself could be tuned by changing the concentrations and sequences of the soluble linker strands $[12,19,20]$. Furthermore, as we discuss below, linker-mediated assembly could in principle enable the programming of large sets of specific interactions using 
considerably fewer unique sequences than required by systems interacting through direct hybridization.

Previous experimental studies have shown that linkers can indeed induce assembly of simple mixtures of DNAcoated nanoparticles. For example, work by Gang and co-workers showed that linkers must be flexible and exceed a minimum concentration per particle to induce crystallization $[20,21]$. Other work by Mirkin and coworkers showed that palindromic linkers can direct assembly of close-packed unary crystals, whereas divalent linkers can assemble binary lattices [19]. While both studies make important contributions to understanding the state space of assembly at low linker concentrationswhen do crystals form-they do not explore the phase behavior that emerges at high concentration. Moreover, a number of other important questions remain open: How is the pair-interaction free energy determined by the experimental inputs, such as linker sequences and concentrations? How can the interactions be modeled and thus programmed? What are the practical limits of linker-based systems? And can we go beyond simple mixtures to realize the original goal of programmable self-assembly: that user-prescribed structures can be assembled from complex mixtures of building blocks through rational design of their interactions?

In this article, we combine experiments and theory to explore the interactions and phase behavior that emerge when binding between DNA-grafted colloidal particles is encoded in soluble linker molecules. Our experiments reveal a rich phase diagram containing two previously unknown regions: (1) a reentrant melting transition that occurs upon increasing linker concentration and (2) a linker concentration at which coexistence between gas and solid is stable over a wide range of temperatures. We show that the phase boundaries separating gas and solid can be tuned by adjusting the grafting density, linker sequence, and concentration, and also demonstrate that a number of competing linker sequences can coexist in the same solution without interfering with one another, suggesting that it might be possible to encode hundreds of interactions simultaneously. Lastly, we develop a statistical-mechanical model that captures the unique phase behavior that we observe quantitatively and derive closed-form equations to predict the pair interaction between colloids from experimental inputs. The combination of our experimental findings and new approaches to modeling shows that we can predict and thus program the interactions required to direct assembly of prescribed aperiodic structures.

\section{RESULTS AND DISCUSSION}

\section{A. Linker-mediated phase behavior}

Our experimental system consists of a binary mixture of micrometer-diameter colloidal particles coated with single-stranded DNA [16]. Each particle species bears
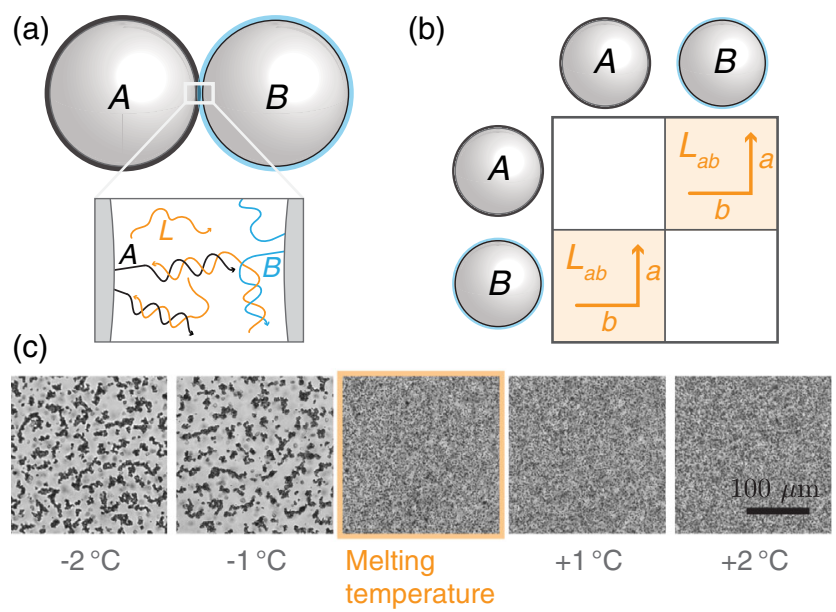

FIG. 1. Linker-mediated binding. (a) Our experimental system is comprised of three DNA sequences: Strands $A$ and $B$ are grafted to $1-\mu \mathrm{m}$-diameter colloidal particles, and strands $L_{a b}$, which bind $A$ to $B$, are dispersed in solution. (b) These three DNA sequences produce a symmetric interaction matrix, in which the linker encodes the pair interaction between particles $A$ and $B$. (c) The resulting phase behavior is temperature dependent: The system phase separates upon decreasing temperature, as shown by optical micrographs. We define the melting temperature $\left(T_{m}\right)$ as the temperature at which $50 \%$ of the particles are unbound. Experiments are for a 19-nucleotide linker at a concentration of $1 \mu \mathrm{M}$; the melting temperature is $43^{\circ} \mathrm{C}$.

65-base-long, single-stranded DNA oligonucleotides which have a 54-thymine spacer and a unique 11 nucleotide (nt) "sticky end" called either $A$ or $B$. The sticky ends are not directly complementary but can be linked together by single-stranded oligonucleotides dissolved in solution, which are half complementary to $A$ and half complementary to $B$ [Fig. 1(a)]. We call these strands "linkers."

The linker-dependent interactions can be represented in a symmetric matrix [Fig. 1(b)], where each element of the matrix is encoded by a single linker sequence (e.g., $L_{a b}$ specifies the interactions between $A$ and $B$ ). As with binding due to direct hybridization, our linker-mediated interactions are temperature dependent: The particles aggregate when cooled and disaggregate when heated [Fig. 1(c)]. We characterize the phase behavior of our system using the melting temperature $T_{m}$, which we define as the temperature at which half of the particles are completely unbound [13]. See Supplemental Material Sec. S1 for experimental details [22].

The concentration-dependent phase behavior that we find is unexpectedly rich, featuring three distinct regions upon increasing linker concentration (Fig. 2). At the lowest linker concentrations, particles do not aggregate even at room temperature [Fig. 2(a), region I]. At intermediate linker concentrations, the particles aggregate at low temperatures and disaggregate when heated [Fig. 2(a), region II]. Within this region of the phase diagram, the temperature at which the particles disassociate $T_{m}$ increases 


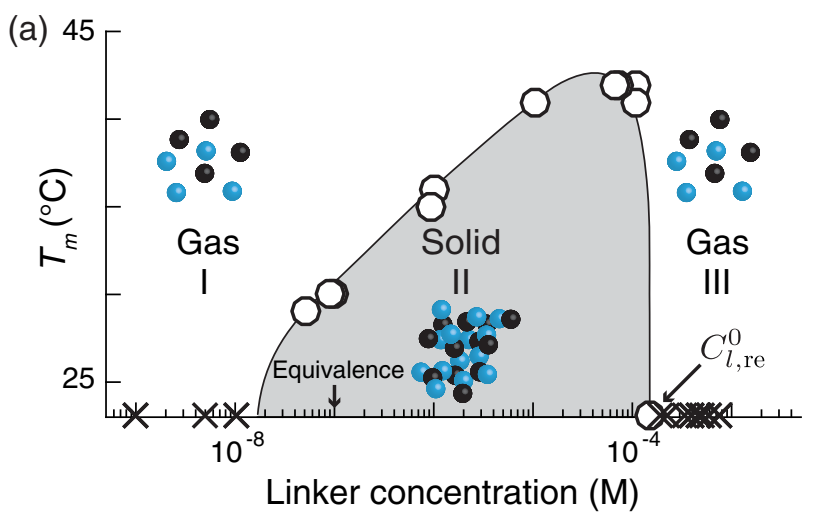

(b)

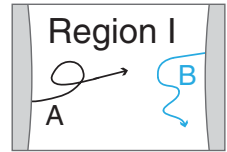

$A+B \leftrightharpoons A+B$

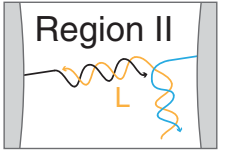

$A+L+B \leftrightharpoons A L B$

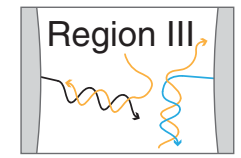

$A+2 L+B \leftrightharpoons A L+B L$
FIG. 2. Phase behavior of linker-mediated assembly. (a) The temperature-linker concentration phase diagram is characterized by three distinct regimes: a gas phase at low linker concentrations (region I), a solid-gas transition at intermediate linker concentrations (region II), and a reentrant gas phase at the highest linker concentrations (region III). Circles show measurements of the melting temperatures at different linker concentrations; $x$ 's show samples that are gas at all temperatures. We define the reentrant concentration $C_{l, \text { re }}^{0}$ as the linker concentration above which the solid melts. The arrow labeled "equivalence" shows the linker concentration at which there is one linker molecule per grafted molecule in the system. Data are for the 17-nt linker and particles with a DNA grafting density of $2000 \mathrm{DNA} / \mu \mathrm{m}^{2}$. The curve is a guide to the eye. We hypothesize that the phase behavior in (a) results from the molecular-scale reactions shown in (b).

monotonically with increasing linker concentration, increasing by roughly $10-15^{\circ} \mathrm{C}$ upon increasing the linker concentration from about $100 \mathrm{nM}$ to $100 \mu \mathrm{M}$. Above an even higher linker concentration the particles fail to aggregate completely over the entire experimental temperature range [Fig. 2(a), region III]. We call the linker concentration above which particles fail to aggregate the "reentrant concentration" $C_{l, \mathrm{re}}^{0}$.

We can understand the observed phase behavior qualitatively by considering the molecular-scale reactions that give rise to interactions between colloidal particles [Fig. 2(b)]. At the lowest linker concentrations, there are too few linkers to stabilize bridges linking particles together. At intermediate linker concentrations, linkers can form bridges between particles via the molecular-scale reaction $A+L+B \leftrightharpoons A L B$. Under these conditions, the melting temperature increases upon increasing linker concentration, since increasing the amount of linker shifts the local equilibrium toward the bridged conformation $A L B$. At the highest linker concentrations, we suspect that every grafted strand is bound to its own linker. Since the linkers cannot bind to other linkers, the particles become effectively passivated against assembly. We note that the state with two half-bridges $A L$ and $B L$ has the same total number of base pairs as the bridged state $A L B$; thus, we suspect that the solid phase within region II is actually stabilized by the entropy of the free linker strands dispersed in solution [23].

The generic phase diagram that emerges illustrates two important features of linker-mediated selfassembly: (1) There is a wide "dynamic range" of linker concentrations-spanning roughly 4 orders of magnitude — over which the melting temperature (and thus, the interaction strength) can be tuned, and (2) there is a linker concentration above which colloids cannot selfassemble, irrespective of the temperature. To explore these two features more fully, we perform similar experiments for different grafting densities and linker lengths (i.e., molecular binding affinities). In both cases, we find qualitatively similar phase behavior with respect to increasing linker concentration as before-the melting temperature first increases and then decreases rapidly-but the melting temperatures and boundaries between regions I, II, and III change (Fig. 3).

We find that increasing the linker affinity increases the melting temperature within region II, but it does not

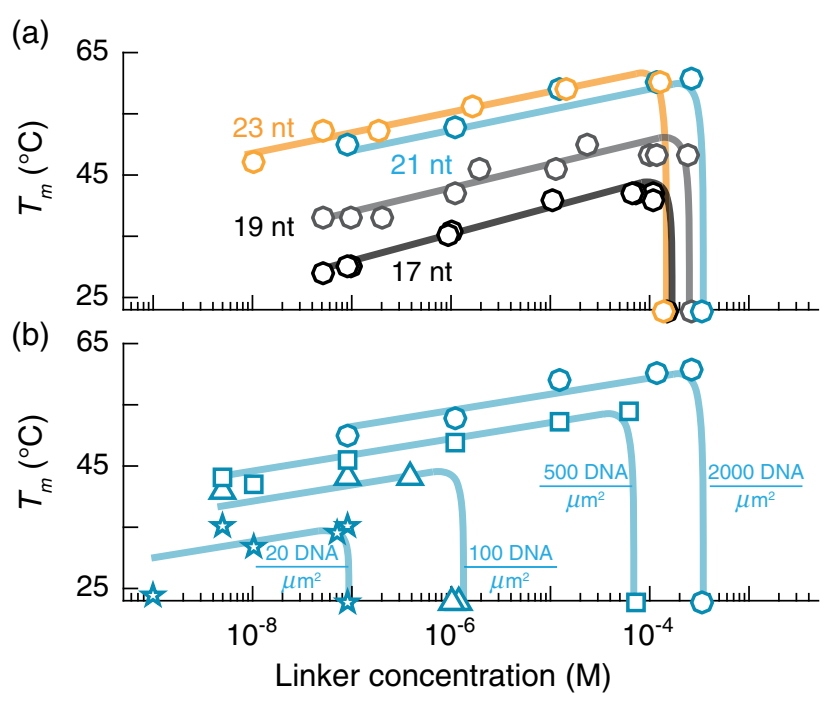

FIG. 3. Effects of linker affinity and grafting density on the phase behavior. (a) Experimental measurements of the melting temperature as a function of the linker concentration for four linkers having different lengths $n$ : $17 \mathrm{nt}$ (black), $19 \mathrm{nt}$ (gray), $21 \mathrm{nt}$ (blue), $23 \mathrm{nt}$ (orange). All linkers are symmetric and $(n-1) / 2$ bases of each linker are complementary to each grafted strand. (b) Measurements of the melting temperature as a function of the concentration for the 21-nt linker for four different grafting densities: 2000 (circles), 500 (squares), 100 (upward triangles), and 20 (stars) DNA strands per $\mu \mathrm{m}^{2}$. The grafting densities of $A$ and $B$ are equal to one another. The reentrant concentrations are shown as points intersecting the linker-concentration axis and have a precision of roughly a factor of 2 . Lines in (a) and (b) are guides to the eye. 
affect the reentrant concentration appreciably. We measure the melting temperature as a function of the linker concentration for four linkers having different lengths: 17, 19, 21 , and 23 nucleotides. We find that the melting temperature increases monotonically with increasing linker affinity, changing by about $20^{\circ} \mathrm{C}$ between 17 and $23 \mathrm{nt}$ [Fig. 3(a)]. The reentrant concentration, however, remains unchanged: All four linkers fail to aggregate above linker concentrations of roughly 200-300 $\mu \mathrm{M}$ [Fig. 3(a)].

Changing the grafting density, in contrast, has two effects: Both the melting temperature and the reentrant concentration decrease with decreasing grafting density. Here, we prepare different batches of colloids $A$ and $B$ with grafting densities ranging from roughly 20 to $2000 \mathrm{DNA} / \mu \mathrm{m}^{2}$ and measure their melting temperatures as a function of the linker concentration $C_{l}^{0}[$ Fig. 3(b)]. As grafting density decreases, we find that the melting temperature decreases monotonically by roughly $15^{\circ} \mathrm{C}$ over the range we explore. We also find that the reentrant concentration decreases with decreasing grafting density, shifting from roughly $300 \mu \mathrm{M}$ to $90 \mathrm{nM}$ (about a factor of $10^{4}$ ) over the 100-fold change in grafting density explored in our experiment, hinting at a squared dependence between the reentrant concentration and grafting density.

Returning to our two observations concerning selfassembly from above, these experiments demonstrate that we can further increase the range over which we tune the interaction strength by adjusting the linker affinity, in addition to the linker concentration. The qualitative trends relating affinity and grafting density to the melting temperature are consistent with our molecular-scale description of the phase behavior: Increasing linker affinity or grafting density (i.e., the concentrations of $A$ and $B$ ) should shift the equilibrium toward the bridged conformation $A L B$, stabilizing the solid phase and increasing the melting temperature. We also find that we can adjust the range of workable linker concentrations by adjusting the grafting densities: Higher grafting densities lead to higher reentrant concentrations and thus yield a wider dynamic range.

We highlight that the reentrant transition does not occur at linker concentrations for which there is one linker for each grafted strand in the system. We call this concentration the "equivalence point" [labeled "equivalence" in Fig. 2(a)]. Indeed, for a grafting density of $2000 \mathrm{DNA} / \mu \mathrm{m}^{2}$ and a total particle volume fraction of $0.5 \%$, there are roughly 2000 linkers for each grafted DNA strand at the reentrant concentration that we find in experiment [Figs. 2(a) and 3(a)]. Furthermore, if the reentrant transition were determined exclusively by the number of grafted strands per particle, we would expect the reentrant concentration to scale linearly with the grafting density. In contrast, we find that the reentrant concentration exhibits a squared dependence on the grafting density. These two observations rule out the explanation that the reentrant transition is governed simply by the ratio of linkers to grafted strands.

\section{B. Mean-field theory}

To confirm our physical picture from above and to develop a quantitative link relating the experimental parameters to the effective interactions that emerge between colloids, we develop a mean-field theory of linker-mediated binding. Modeling the phase behavior involves two steps: (1) First we develop a model to relate the sequence, concentration, and grafting density to the multivalent free energy per particle, and (2) we relate that free energy per particle to the phase behavior of the system as a whole.

We adapt a recent approach developed in Ref. [24], which models interactions that result from multimeric ligand-receptor complexes to calculate the free energy per particle in the gas and solid phases. Briefly, this involves computing the equilibrium densities of the different molecular species-bridges, half-bridges ( $A L$ or $B L)$, and unbound strands - and relating those densities to the free energy of multivalent binding between two particles [24]. In our specific case, we compute the surface densities of half-bridges $\rho_{1}$ and full bridges $\rho_{b}$ from equations of local chemical equilibrium [14,25], starting from the densities of unhybridized grafted strands $A$ and $B\left(\rho_{A}\right.$ and $\left.\rho_{B}\right)$, and the concentration of free linkers in solution $\left(C_{l}\right)$ :

$$
\begin{aligned}
& \rho_{1}(h)=\rho_{A}(h) \frac{C_{l} e^{-\beta \Delta G_{0} / 2}}{C^{\circ}}, \\
& \rho_{b}(h)=\rho_{A}(h) \rho_{B}(h) \frac{C_{l}}{C^{\circ}} e^{-\beta \Delta G_{0}} K(h), \\
& \rho_{A}(h)=\rho_{B}(h)=\Psi-\rho_{1}(h)-\rho_{b}(h),
\end{aligned}
$$

where $\Psi$ is the surface density of grafted strands, $\beta=$ $1 / k_{B} T$ is the reciprocal of the thermal energy $k_{B} T, \Delta G_{0}$ the sum of the hybridization free energies of pairing $A$ with $L$ and $B$ with $L, C^{\circ}=1 \mathrm{M}$ is the reference concentration at which $\Delta G_{0}$ is defined, and $K(h)$ is an effective area accounting for the configurational costs associated with bridge formation $[25,26]$. For simplicity, we assume that the hybridization free energies of reacting $A$ with $L$ and $B$ with $L$ are the same. The general case is presented in the Supplemental Material Sec. S2 [22].

We add a new element to our mean-field theory to capture effects due to the fixed anchor points of grafted DNA strands. Because the grafted molecules are immobilized on the surface of particles, their densities are spatially nonuniform in the contact region between two particles. Therefore, we evaluate the equilibrium densities locally. We define $h$ to be the local distance between the particles' surfaces: $h$ is a minimum along the line of centers and extends to the maximum separation distance at which bridges can form. Since $\rho_{l}(h)$ and $\rho_{b}(h)$ are coupled for all values of $h$ by the concentration of free linkers $C_{l}=$ $C_{l}^{0}-\rho\left[n_{1}+\left(n_{b} / 2\right)\right]$, where $\rho$ is the density of colloids, the numbers of half-bridges $n_{1}$ and bridges $n_{b}$ per particle are calculated self-consistently by integrating $\rho_{1}$ and $\rho_{b}$ over 
the particles' surfaces. We highlight that previous theories do not include these effects [24,27]. See Supplemental Material Sec. S2 for details [22].

Next, we derive the phase boundaries by equating the chemical potential of the gas to the chemical potential of the solid. We model the solid phase as a cluster of particles with coordination number $Z$. As is usually done for particles whose interaction range $\delta$ is much smaller than their radius $(\delta \ll R)$, we use a cell model in which particles in the solid phase are assumed to move independently within a volume $v_{f}[28,29]$. Following the same arguments as Ref. [29], we find that the melting temperature is given by $\Delta f_{\text {coll }} / k_{B} T_{m}=\log \left(\rho v_{f}\right)+1$, where $\Delta f_{\text {coll }}$ is the free energy per particle. It should be noted that the previous expression is derived using pairwise square-well potentials with an attractive well depth equal to $\Delta f_{\text {coll }}$ and interaction range equal to $\delta$ with $v_{f}=(\delta / 2)^{3}$ [28]. In the present case, $\Delta f_{\text {coll }}$ is not strictly pairwise since the concentration of free linkers $C_{l}$ depends on the distances between all interacting particles. However, we ignore this effect.

Our mean-field theory reproduces the phase diagram that we find in our experiments. Figure 4(a) shows a comparison between our experimentally measured melting temperatures and the predictions of our mean-field theory for the 19-nt linker and a grafting density of $2000 \mathrm{DNA} / \mu \mathrm{m}^{2}$. Both agree quantitatively above linker concentrations of roughly $10 \mathrm{nM}$ : The melting temperature increases logarithmically from 40 to $50^{\circ} \mathrm{C}$ upon increasing linker concentration up until roughly $300 \mu \mathrm{M}$, at which point the melting temperature plummets. We note a discrepancy between theory and experiment at the lowest linker concentrations $(<10 \mathrm{nM})$. Here we find suspensions that are always disaggregated in experiment, whereas our theory predicts a melting temperature that decreases more softly. We hypothesize that this disagreement is due to kinetic limitations and a difficulty for our system to equilibrate on experimental timescales.

Examining the number of multimeric complexes predicted by our mean-field theory confirms our molecularscale description of the nature of the transitions between regions I, II, and III. Figure 4(b) shows the number of bridges and half-bridges $(A L$ or $B L)$ in the contact region between two particles within the solid phase as a function of linker concentration for two distinct pathways through the phase diagram. Following a path at constant temperature [indicated by the blue arrow in Fig. 4(a)], we find that the number of bridges in equilibrium is $<1$ per contact at linker concentrations below roughly $1 \mathrm{nM}$ : There are too few bridges to stabilize the solid phase. Upon increasing the linker concentration, the number of bridges $A L B$ increases monotonically until linker concentrations of roughly $1 \mu \mathrm{M}$. Within this intermediate region, bridges greatly outnumber half-bridges. As the linker concentration is increased further, the number of bridges decreases as
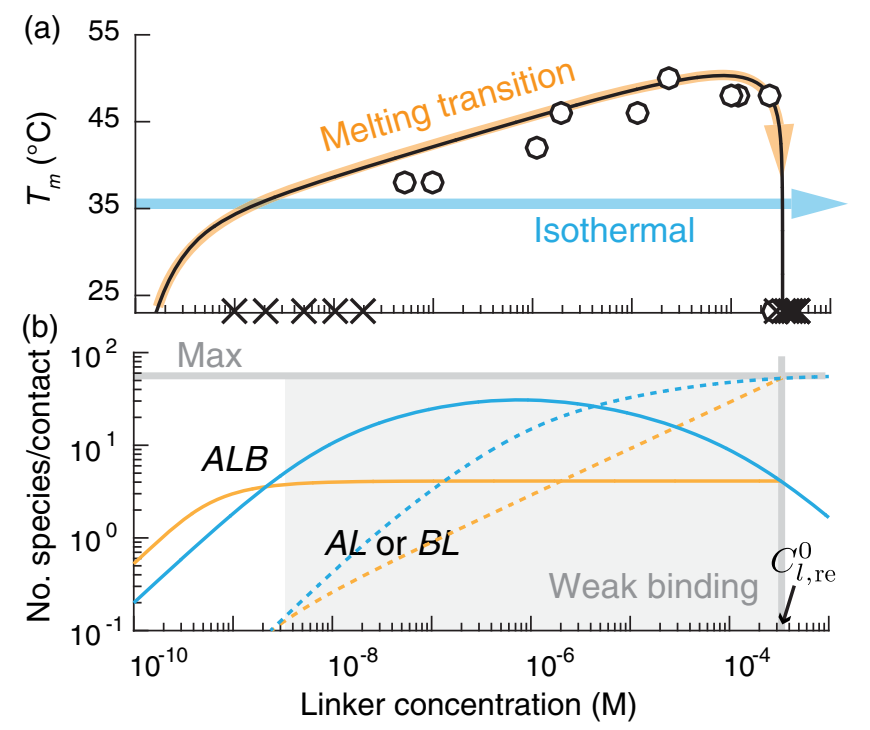

FIG. 4. Predictions of the melting temperature $T_{m}$ and number of bound molecular species per contact between particles versus linker concentration. (a) The full theory predicts a phase diagram that matches experimental measurements (points) quantitatively above linker concentrations of roughly $10 \mathrm{nM}$. (b) Predictions of the number and type of bound molecular species-either bridges (solid lines) or half-bridges (dashed lines)—between a pair of interacting particles help explain our observations. Orange curves show the number of molecular species at the melting transition [the orange path in (a)]; blue curves show the number of bridge and half-bridge species at a fixed temperature: $T=35^{\circ} \mathrm{C}$ [the blue path in (a)]. Linker concentrations with a constant number of bridges at the melting transition correspond to the weak-binding limit. The horizontal gray line shows the maximum number of possible bound species; the vertical gray line shows the reentrant concentration.

half-bridges take over and eventually saturate nearly all grafted molecules at the reentrant concentration.

Taking an alternative path through the phase diagram, one which follows the phase boundary, highlights another unique feature of our system: The number of bridges at the melting transition is roughly constant above a certain linker concentration (again about $>10 \mathrm{nM}$ ). Following the path indicated by the orange arrow in Fig. 4(a), our meanfield theory predicts that there are roughly four bridges per contact for all linker concentrations above $10 \mathrm{nM}$ and below the reentrant concentration [Fig. 4(b)]. This prediction hints at the possibility that our measurements and predictions of the phase boundary occur in the so-called "weak-binding limit," in which the free energy per particle is approximated by the average number of bridges per particle multiplied by the thermal energy $\Delta f_{\text {coll }} \approx-\left\langle n_{b}\right\rangle k_{B} T / 2[12,14,30]$.

\section{Weak-binding limit: Melting temperature}

We explore the possibility that our experiments might be described by a weak-binding-limit approximation. Specifically, we take the limit of our full theory as 
$\Delta G_{0} \rightarrow \infty$, resulting in a compact analytic expression for $T_{m}$ :

$$
\frac{\Delta H_{0}}{k_{B} T_{m}}-\frac{\Delta S_{0}}{k_{B}}=\ln \left[\frac{-Z \pi R v_{0}}{2\left[\ln \left(\rho v_{f}\right)+1\right]} \frac{\Psi_{A} \Psi_{B} C_{l}^{0}}{C^{\circ}}\right],
$$

where $\Delta H_{0}$ is the enthalpy change of hybridization, $\Delta S_{0}$ is the entropy change of hybridization, $R$ is the particle radius, $\Psi_{i}$ is the grafting density of particle species $i$, and $v_{0}$ is a microscopic interaction volume defined as $v_{0}=\int K(h) d h$ (see Supplemental Material Sec. S3 for details [22]). Examining Eq. (1), we find that the free energy of hybridization at the melting transition $\Delta G_{0}\left(T_{m}\right)=\Delta H_{0}-T_{m} \Delta S_{0}$, which depends on the linker sequence, is balanced by an entropic term, which has a logarithmic dependence on the grafting densities and linker concentration - the other two independent variables in our experiment.

To test the predictions of our weak-binding-limit model, we measure the melting temperatures for hundreds of unique combinations of linker lengths, linker concentrations, and particle grafting densities for the case where $\Psi_{A}=\Psi_{B}$. The data that we find show melting temperatures ranging from roughly 25 to $65^{\circ} \mathrm{C}$, reentrant concentrations spanning from $1 \mathrm{nM}$ to $300 \mu \mathrm{M}$, and follow the same basic trends as before: The melting temperature decreases with decreasing linker concentration, decreasing grafting density, and decreasing linker length, whereas the reentrant concentration decreases only with decreasing grafting density [Fig. 5(a)].

Remarkably, we find that all $200+$ measurements of the melting temperature collapse to a single master curve when rescaled according to Eq. (1). Figure 5(b) shows the hybridization free energy evaluated at the melting temperature versus the right-hand side of Eq. (1), as well as the predictions from the weak-binding-limit model (the $y=x$ line) and the full theory. We find that all data collapse to a narrow band which falls just below the weakbinding predictions. The spread in the rescaled data reflects a variation in the melting temperatures of roughly $\pm 1.5^{\circ} \mathrm{C}$, which is consistent with our experimental precision of roughly $1{ }^{\circ} \mathrm{C}$. The offset indicates a minor discrepancy between the weak-binding model and our experimental measurements of about $3^{\circ} \mathrm{C}$. We note that the full theory is also offset slightly from the weakbinding limit, though by a smaller extent, and that the offset grows at the lowest grafting densities and linker concentrations explored. Here we expect the weakbinding approximation to break down since the depletion of free linkers-an effect not considered in the weakbinding limit—becomes important.

\section{Weak-binding limit: Reentrant concentration}

Next, we explore the dependence of the reentrant concentration on the grafting density in the weak-binding
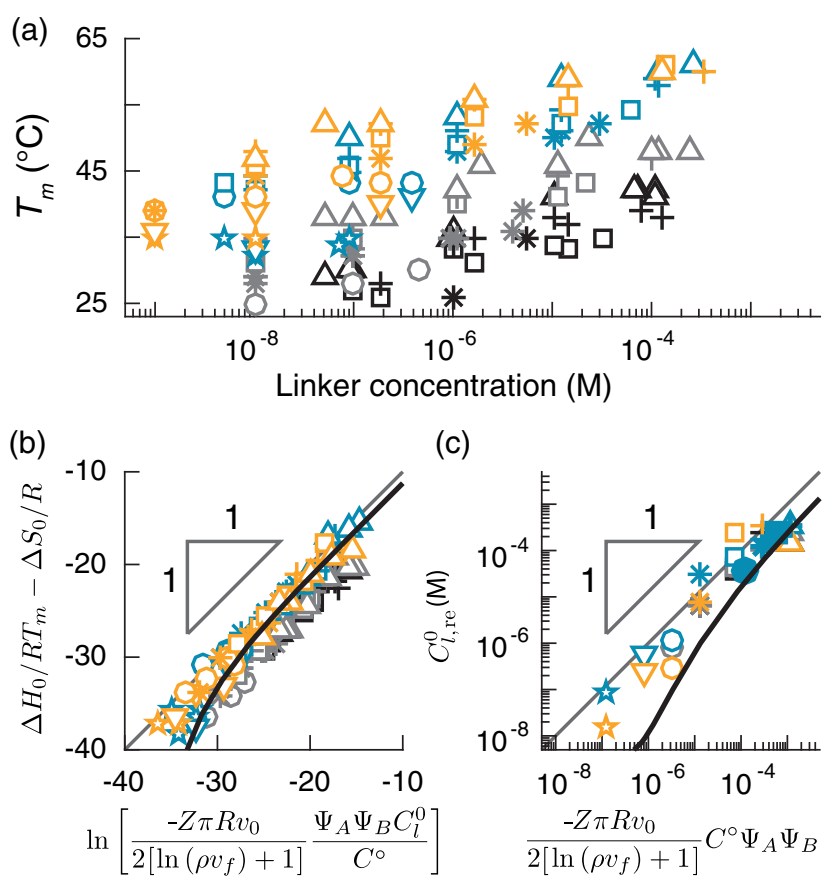

(c)

FIG. 5. Melting temperatures and reentrant concentrations collapse onto master curves. (a) shows roughly 200 measurements of the melting temperature (points) for different combinations of linker concentration, linker length, and grafting density. Color indicates linker length: $17 \mathrm{nt}$ (black), $19 \mathrm{nt}$ (gray), $21 \mathrm{nt}$ (blue), and $23 \mathrm{nt}$ (orange). Symbols indicate grafting density: 2000 (upward triangle), 1000 (plus), 500 (square), 200 (asterisk), 100 (circle), 50 (downward triangle), and 20 (star) DNA strands per $\mu \mathrm{m}^{2}$. (b) The same data collapse when rescaled according to Eq. (1). (c) The measured reentrant concentrations collapse and exhibit a power-law dependence on the grafting density $\Psi$, as given by Eq. (2). Filled symbols correspond to unequal grafting densities. The solid black curves in (b),(c) show predictions of the full theory. The black curve in (b) is generated by rescaling the full-theory predictions at intermediate concentrations below $C_{l, \mathrm{re}}^{0}$.

limit. Specifically, we take the limit of our full theory when both the binding is weak and the concentration of linkers is large. Here we find the following expression (see Supplemental Material Sec. S3 for details [22]):

$$
C_{l, \mathrm{re}}^{0}=\frac{-Z \pi R v_{0}}{2\left[\ln \left(\rho v_{f}\right)+1\right]} C^{\circ} \Psi_{A} \Psi_{B}
$$

We see immediately that this expression for the reentrant concentration makes two important predictions: (1) It does not depend on the hybridization free energy and thus does not depend on the linker sequence, and (2) it scales as the product of the grafting densities of particles $A$ and $B$; higher grafting densities yield higher reentrant concentrations. Both of these features are consistent with our observations from before [Fig. 3], and the scaling of the reentrant concentration with $\Psi_{A} \Psi_{B}$ is reminiscent of the squared dependence we saw previously. 
To test the predictions of Eq. (2), we perform two types of experiments: one in which we decrease the grafting densities of both particle species together from 2000-20 DNA $/ \mu \mathrm{m}^{2}$ for each of the four linkers and another in which we hold the grafting density of particle $A$ at $2000 \mathrm{DNA} / \mu \mathrm{m}^{2}$ and decrease the grafting density of particle $B$ for a single linker (19 nt). In both cases, we measure the reentrant concentration to a precision of roughly a factor of 2 .

We find that our measurements of the reentrant concentration again collapse well when plotted against the predictions of Eq. (2), confirming the dependence on the grafting densities. In both cases-either equal grafting densities or mixed grafting densities-we observe a scaling of the reentrant concentration that goes as the product of the two densities over a range spanning roughly 4 orders of magnitude [Fig. 5(c)]. The fact that our data collapse so well confirms the prediction that the reentrant concentration depends not on the grafting density alone, but on the product of the grafting densities of the two particles. Furthermore, these predictions tell us directly the maximum linker concentration that we can use for a selfassembly experiment: It must be less than the reentrant concentration in order for the particles to assemble. The predictions of the full theory show a similar scaling for grafting densities above 50 strands $/ \mu \mathrm{m}^{2}$.

The weak-binding-limit predictions of the melting temperatures and reentrant concentrations [Eqs. (1) and (2)] agree quantitatively with our experimental measurements and provide indispensable tools for programming selfassembly. Specifically, they provide simple closed-form analytic expressions that predict the melting temperature and reentrant concentration from the experimental inputs: linker sequence, linker concentration, and grafting density. Returning to our original motivation of fully addressable self-assembly, Eq. (1) can be used to choose combinations of linker sequences and concentrations that would match the melting temperatures (and thus, binding affinities) of dozens of pairs of interacting particles; Eq. (2) can be used to prepare DNA-coated colloids with sufficiently high grafting densities such that the reentrant concentration is higher than the intended linker concentrations.

\section{Combining multiple linkers}

Thus far, we have considered a situation in which two particle species interact with one another via a single linker sequence. However, we ultimately aim to use combinations of many linker sequences to specify the complex interaction matrix between many different particle species simultaneously. For instance, to self-assemble a modest structure formed from only a few same-size particles-like the 19-particle dipyramid shown in Fig. 6(a)-requires programming $>50$ specific interactions. Within the framework we propose, each particle species must then bind to multiple unique linker sequences-one for each pair interaction - which requires that some individual particles will need as many as 12 different linker sequences to specify their interactions with their neighbors in the final structure. This constraint begs the question: Does adding multiple linker sequences, some of which bind to the same particle species and thus the same grafted sequence, interfere with binding or compromise the validity of our models developed above? For instance, does adding a linker sequence which binds $A$ to $C$ interfere with the binding of a linker which binds $A$ to $B$ ?

We investigate the cross talk between different linker sequences by designing a slight variant on our previously described experiments. Specifically, we modify our 19-nt linker so that it binds only to one grafted strand and replace the other half of the linker's bases with a series of inert thymines. Thus, instead of forming bridges, our modified linkers can form only half-bridges, which inhibit assembly. We call these modified linkers "competitors." In the context of complex self-assembly, the competitors act like other linker species that would bind particles $A$ or $B$ to other particle types in the same solution [Fig. 6(b)]. Indeed, we design two of these: $L_{a}$ which binds to particle $A$ but not particle $B$, and $L_{b}$ which binds to particle $B$ but not $A$. We then mix these competitors together with our active linker $L_{a b}$ - the only linker sequence that can form bridges - and investigate the effect of increasing competitor concentrations on the melting temperature of our colloidal suspension.

If the thermodynamics of linker-mediated binding were unaffected by the presence of other linkers in solution, we would not expect to observe a change in the melting temperature upon the addition of the competitors. Figure 6(c) shows the change in melting temperature of the binary mixture of colloids $A$ and $B$ upon addition of a single competitor species $L_{a}$ at different concentrations of the active linkers. We find that up to quite high concentrations of the competitor, the melting temperature is independent of the amount of competitor added [Fig. 6(c)], even when the competitor is added in 100-fold excess of the active linker (see, for example, the 100-nM active linker and $10-\mu \mathrm{M}$ competitor). Indeed, up to competitor concentrations of roughly $10 \mu \mathrm{M}$, we find that the melting temperature is constant for active linker concentrations ranging from $100 \mathrm{nM}$ to $100 \mu \mathrm{M}$. Above this concentration, the melting temperature decreases, and the particles eventually melt by competitor concentrations of a few hundred $\mu \mathrm{M}$. This behavior is independent of the amount of active linker $L_{a b}$ added to the suspension: We explore $100 \mathrm{nM}, 1,10$, and $100 \mu \mathrm{M}$ and find the same behavior in all cases. Predictions from the full theory agree quite well with our experimental measurements for all but the lowest linker concentration.

A more stringent test of the competition between different linker species is to add both competitor linkers $L_{a}$ and $L_{b}$ simultaneously. Here we find similar qualitative 
(a)

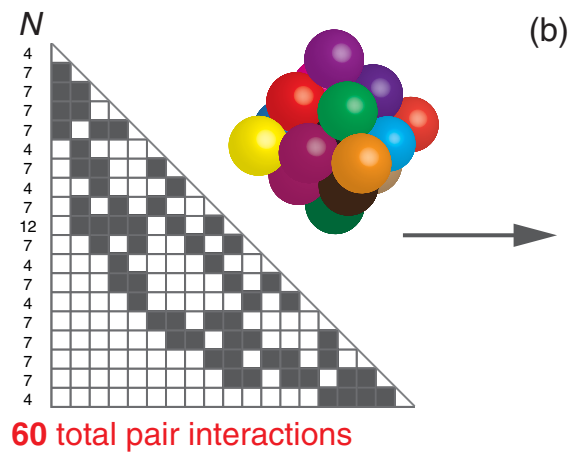

(c)

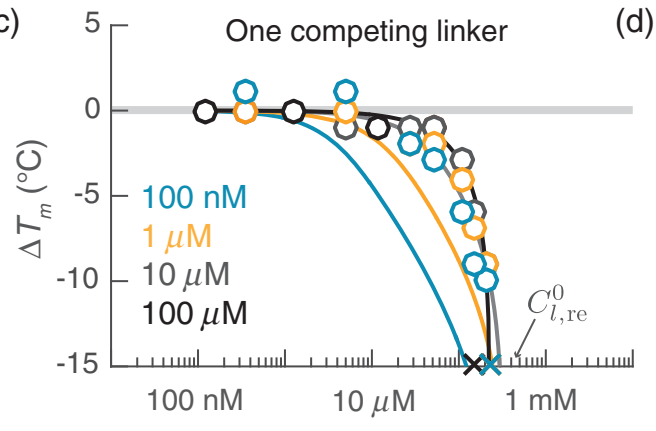

Competitor concentration (b)

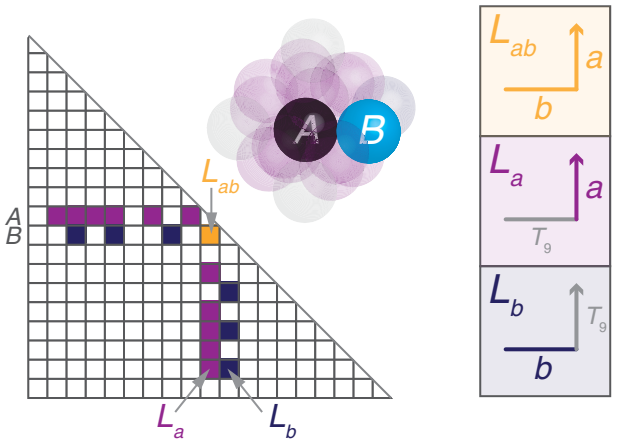

(d)

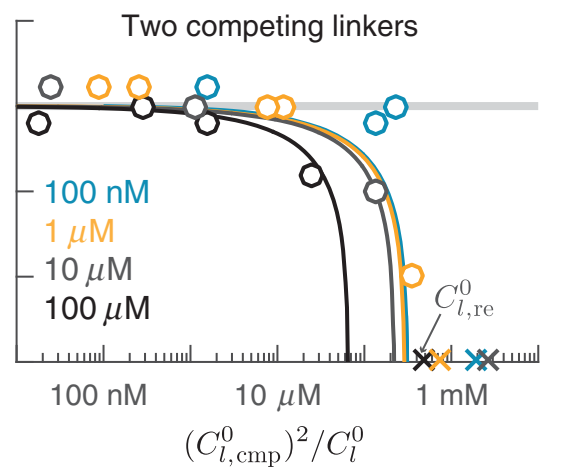

FIG. 6. Effects of multiple competing linker sequences on pair interactions. (a) Fully addressable self-assembly of a 19-particle dipyramid requires specifying 60 independent pair interactions shown in the interaction matrix with some particles needing up to 12 pair interactions. The number of specific interactions per particle $N$ is shown to the left of the matrix. (b) We test the feasibility of this design by devising a simplified experimental system consisting of two particle species, $A$ and $B$, and three linker sequences $L_{a b}, L_{a}$, and $L_{b}$. The monovalent linkers $L_{a}$ and $L_{b}$ compete with the divalent linker $L_{a b}$ and simulate the role of other linkers binding $A$ and $B$ to other particle species. (c) Measurements of the change in melting temperature of particles $A$ and $B$ for increasing concentrations of a single competing linker $L_{a}$ (points). Colors indicate different concentrations of $L_{a b}: 100 \mu \mathrm{M}$ (black), $10 \mu \mathrm{M}$ (gray), $1 \mu \mathrm{M}$ (orange), and $100 \mathrm{nM}$ (blue). (d) Measurements of the change in the melting temperature for two competing linkers with $C_{l, \mathrm{cmp}}^{0} \equiv C_{l, A}^{0}=C_{l, B}^{0}$ versus $\left(C_{l, \mathrm{cmp}}^{0}\right)^{2} / C_{l}^{0}$. Curves in (c),(d) show predictions from the full theory; $\boldsymbol{x}$ 's show experimental concentrations at which the particles fail to aggregate. All particles have a surface density of $2000 \mathrm{DNA} / \mu \mathrm{m}^{2}$, and the reentrant concentration is indicated by the arrow. The reentrant concentration indicated by $C_{l, \mathrm{re}}^{0}$ in (c),(d) is calculated by the full theory in the absence of competitors.

behavior: The melting temperature remains unchanged at low competitor concentrations and then decreases above some threshold until the particles no longer aggregate. Figure 6(d) shows the change in melting temperature versus increasing competitor concentration rescaled by $\left(C_{l, \mathrm{cmp}}^{0}\right)^{2} / C_{l}^{0}$, where $C_{l, \mathrm{cmp}}^{0}$ is the concentration of $L_{a}$ and $L_{b}$ (we keep the concentrations of both competitors the same). Below a rescaled concentration of roughly $10 \mu \mathrm{M}$, the melting temperature does not change. Above this threshold, the melting temperature decreases with increasing competitor concentration, with a transition that appears to depend weakly on the active linker concentration: The melting temperature of the highest active linker concentration $(100 \mu \mathrm{M})$ decreases by roughly $5{ }^{\circ} \mathrm{C}$ at a scaled competitor concentration of about $10 \mu \mathrm{M}$, whereas the melting temperature of the $100-\mathrm{nM}$ active linker case does not decrease until the scaled competitor concentration exceeds $>100 \mu \mathrm{M}$. These data are again described accurately by predictions from the full theory. Furthermore, the data also agree well with a high-competitor-concentration scaling limit, which shows that the reentrant concentration should be $\sqrt{C_{l}^{0} C_{l, \text { re }}^{0}}$ (see Supplemental Material Sec. S3 for details [22]).

At first blush, the results we find-that adding a (10-100)-fold excess competitor does not interfere with the assembly of $A$ to $B$ via linker $L_{a b}$-are counterintuitive. However, the predictions of our model provide an explanation. The change in the phase behavior that we observe in our competitor experiments can be understood as a competition between half-bridges, which could be formed by either active linkers or competitors, and bridges, which can be formed only by active linkers. The observation that the melting temperature is unchanged by modest concentrations of competitor suggests that bridges are considerably more thermodynamically stable than half-bridges. Indeed, returning to Fig. 4(b) we see that bridges outnumber halfbridges up until linker concentrations of roughly $1 \mu \mathrm{M}$ and that half-bridges do not occupy a majority of grafted sites until concentrations above $10 \mu \mathrm{M}$. In other words, at low competitor or linker concentrations, the system would 
prefer to form bridges instead of half-bridges, again due to the entropy of the free linkers dispersed in solution (Supplemental Material Sec. S2 [22]). At higher concentrations, the situation changes and half-bridges become more thermodynamically stable than bridges. This same mechanism is responsible for the decrease in melting temperature that we observe in our competitor experiments, as well as for the reentrant melting transition that we observed previously.

Most importantly, our findings demonstrate that multiple linker species can in fact be added together without interfering with one another, provided that the concentrations of linkers are below a threshold value. A back of the envelope estimation shows that linker-prescribed assembly of modest aperiodic structures can be accomplished using our scheme. Given that same-sized spherical particles are able to have at most 12 neighbors [31], there need not be more than 12 linker sequences that bind to the same particle type corresponding to a competitor-to-linker ratio of $11: 1$. Our experimental data show that even a $20: 1$ ratio of competitor to linker does not change the melting temperature appreciably for linker concentrations in the range of $100 \mathrm{nM}$ to $10 \mu \mathrm{M}$. Thus, we conclude that we can indeed prescribe the 60 total pair interactions needed to encode the 19-particle dipyramid in Fig. 6 using 60 unique linker sequences and then match all of their pair-interaction free energies using the theory we develop above.

\section{CONCLUSIONS}

In this work, we show that linker-mediated self-assembly has a number of interesting features and distinct advantages compared to self-assembly of DNA-coated colloids due to direct hybridization. First, many distinct linker sequences can be combined to specify and tune the hundreds of specific interactions needed to encode a prescribed aperiodic structure as the only ground state in a complex mixture of same-sized colloids. Unlike DNA interactions due to direct binding in which every orthogonal pair interaction must be specified by a different grafted sequence and the mutual interaction strengths are hardwired once the particles are synthesized, linker-mediated interactions can be tuned in situ by adjusting the linker concentrations. Furthermore, many specific interactions can be encoded between particles which are each grafted with a single sequence by creating cocktails of many linker sequences in the same solution: one linker sequence per pair interaction. This feature of linker-mediated binding greatly expands the ability to encode the large sets of interactions required for fully addressable assembly.

We highlight the potential to prescribe complex interaction matrices using linkers by considering two different scenarios: (1) in which one wishes to specify and tune every pair interaction independently in a system containing $P$ particle species and (2) in which one wishes to specify the maximum number of interactions given $S$ orthogonal DNA sequences.
In scenario 1 , there are $\left(\begin{array}{l}P \\ 2\end{array}\right)+P=P(P+1) / 2$ total pair interactions: $A$ with $A, A$ with $B, A$ with $C, B$ with $B, B$ with $C$, and so forth. Encoding each one of these interactions independently using direct binding requires one unique grafted sequence per pair interaction, and thus, $P(P+$ $1) / 2$ grafted sequences. In contrast, encoding every possible pair interaction independently between $P$ particle species in a linker-based system requires only $P$ distinct grafted sequences: one grafted sequence per particle, together with $P(P+$ 1) $/ 2$ linker sequences. In scenario $2, S$ orthogonal sequences can be used to specify $S$ pair interactions in the direct-binding case by grafting the $S$ sequences and their complements to particles. In contrast, one can generate $\left(\begin{array}{l}S \\ 2\end{array}\right)=S(S-1) / 2$ unique linker sequences from $S$ total sequences. Thus, it is possible to prescribe up to a factor of $(S-1) / 2$ more unique pair interactions from a finite pool of sequences using linkers as compared to using direct binding.

The enhanced flexibility in linker-mediated binding results from additional degrees of freedom introduced to the system - the molar concentrations of each linker sequence-which modify the interaction free energy per particle in nontrivial ways. Importantly, we show that the influence of these new degrees of freedom can be modeled quantitatively using our mean-field theory and can thus be programmed a priori. We stress that one technical hurdle to assembling prescribed structures from uniformly coated spheres remains: The structures must be assembled in systems containing only one of each particle species. Microfluidics-based methods have been developed to conduct experiments within such constraints [32], but these considerations are beyond the scope of this article.

We also show that the phase behavior of linker-mediated self-assembly is qualitatively different from that of direct binding. Whereas the interaction strengths between DNAcoated colloids due to hybridization of grafted strands increase monotonically with increasing DNA density, the interaction strengths between DNA-coated particles due to linker sequences dissolved in solution are nonmonotonic: The interaction strengths first increase and then decrease upon increasing linker concentration, inducing a reentrant melting transition in the phase diagram. Since this reentrant transition is reproduced by our mean-field theory, which assumes local equilibrium at the molecular scale, we emphasize that the reentrant melting transition should be generic to systems in which assembly is due to weak multivalent binding mediated by free molecules in solution. Indeed, qualitatively similar behavior is observed in a wide range of experimental systems ranging from "squelching" in gene expression [33] to reentrant condensation in proteins [34] and nucleic acids [35] to self-assembly of virus particles [36]. Thus, our model may find applications in a number of other settings. We note that earlier experiments $[12,20,21]$ and theories $[37,38]$ of self-assembly of DNA-coated colloids do not observe this new transition since they focus only on the lowest linker concentrations. 
Finally, while the current study focuses on the phase behavior that emerges in equilibrium, we highlight that linker-based systems could also be used to study nonequilibrium routes to self-assembly. For instance, our demonstration that linker-mediated phase behavior results from the local equilibrium of molecular-scale reactions opens the door to inclusion of complex DNA-based circuits and devices from DNA nanotechnology into colloidal selfassembly, such as catalytic amplifiers, cascaded circuits, and logic gates [39]. The integration of such nonequilibrium devices, which break detailed balance, could yield schemes for error correction, adaptation, and other strategies exploited by biological systems to engineer an astonishing diversity of self-assembling materials [40,41].

\section{ACKNOWLEDGMENTS}

We thank Rees Garmann, Vinothan Manoharan, Eric Weeks, and Michael Hagan for fruitful discussions, and Michael Perlow and Kyra Hamel for their assistance in measuring the hybridization free energies. J. L., G. N. P., and W. B. R. acknowledge support from the National Science Foundation (Grant No. NSF DMR-1710112) and the Brandeis Materials Research Science and Engineering Center (Grant No. NSF DMR-1420382). The work of B. O. and B. M. M. is supported by an Actions de Recherche Concertées (ARC) Grant of the Fédération WallonieBruxelles and by the Fonds De La Recherche Scientifique (FRS-FNRS, Belgium) under Grant No. MIS F.4534.17.

\section{APPENDIX A: MATERIALS}

\section{DNA grafting}

We synthesize DNA-grafted colloidal particles using a technique that physically grafts DNA-conjugated block copolymers to the surface of $1-\mu \mathrm{m}$-diameter polystyrene microspheres (Invitrogen) [16]. Briefly, the terminal hydroxyl ends of a poly(ethylene oxide)-poly(propylene oxide)-poly(ethylene oxide) triblock copolymer (Pluronic F108, BASF) are activated by p-nitrophenyl chloroformate (Sigma-Aldrich). A subsequent reaction with 5'-aminoC6-modified single-stranded DNA oligonucleotides (Integrated DNA Technologies, Inc.) forms a stable carbamate linkage between F108 and DNA. The DNA-conjugated copolymers are then adsorbed to the surface of polystyrene microspheres (Invitrogen) in 10-mM citric acid buffer $(p \mathrm{H}=4)$ and physically grafted by swelling and deswelling the polystyrene cores with toluene. We use a volume of toluene equal to the total volume of polystyrene. Finally, DNA-grafted particles are washed and stored in an aqueous buffer containing 10-mM Tris and 1-mM ethylenediamine tetra-acetic acid (EDTA) $(p \mathrm{H}=8)$ at a colloidal particle volume fraction of roughly $1 \%(\mathrm{v} / \mathrm{v})$. We estimate our maximum DNA density to be 6500 DNA strands per particle and use this value in all calculations presented. We adjust the labeling density from roughly 65 to 6500 strands per particle by diluting the DNA-functionalized F108 with unlabeled F108 before swelling [42].

\section{DNA sequence design and synthesis}

The sequences we use are designed following a prescription described in Ref. [43]. All DNA sequences are designed to minimize formation of stable secondary structures (such as hairpins) and cross talk between noninteracting sequences by ensuring that all three-base codons and their complements are used only once.

Grafted sequences are 65 bases long, single stranded, and consist of an inert poly-dT spacer and functional domain on the 3' end. The poly-dT spacer sets the range of interaction; the sticky end sequences are 11 nucleotides long and provide a unique label for each bead species. All surface-grafted strands are purified by high-performance liquid chromatography.

Soluble linker strands range from 17 to 21 nucleotides in length. A linker of length $n$ has $(n-1) / 2$ nucleotides complementary to each of the grafted strands' sticky ends. All linkers also have one cytosine base between the two binding domains, which acts as a flexible spacer. All linker strands are purified by standard desalting. The specific base sequences we use are given in Supplemental Material Table I [22].

\section{APPENDIX B: METHODS}

\section{Determining thermodynamic parameters}

We measure directly the standard enthalpy change $\Delta H$ and entropy change $\Delta S$ of hybridization for all linkers using ultraviolet (UV) spectrometry, following the procedure outlined in Ref. [24]. Our experimental setup consists of a UV light source (DH-2000-BAL, Ocean Optics), a temperature-controlled cuvette holder (qpod, Quantum Northwest), and a spectrometer (Flame-S-UVVis-ES, Ocean Optics). Briefly, $400 \mu \mathrm{l}$ of sample containing $1 \mu \mathrm{M}$ of each DNA species, $500-\mathrm{mM} \mathrm{NaCl}$, and $1 \mathrm{X}$ Tris-EDTA are loaded into a quartz cuvette (Starna Cells, Inc.) and covered with a layer of mineral oil (SigmaAldrich). The samples are heated to $90^{\circ} \mathrm{C}$ for $20 \mathrm{~min}$ and then cooled to $20^{\circ} \mathrm{C}$ at a rate of $0.5 \%$ min while recording absorption spectra at $0.5^{\circ} \mathrm{C}$ intervals. At each temperature point, the absorption spectrum is averaged over $20 \mathrm{~s}$.

We use the "baseline" method to extract the DNA thermodynamics from our absorption measurements. Briefly, we fit the low- and high-temperature baselines of the temperature-dependent absorption measured at $260 \mathrm{~nm}$ with straight lines $b_{\text {low }}(T)$ and $b_{\text {high }}(T)$ and then compute the fraction of hybridized strands $\theta(T)$ from the absorption data $A(T)$ using $\theta(T)=\left[b_{\text {high }}-A(T)\right] /\left(b_{\text {high }}-b_{\text {low }}\right)$. According to the two-step model of DNA hybridization in which hybridization is represented by the bimolecular reaction $X+L \leftrightarrow X L$, the equilibrium constant $K_{a}=$ $[X L]_{C^{\circ}} /([X][L])$ is related to the standard free energy $\Delta G$ through $K_{a}=e^{-\Delta G / R T}$, where $C^{\circ}$ is a reference 
concentration, $R$ is the gas constant, and $T$ is the temperature. Considering an equimolar mixture of single strands $X$ and $L$ with concentrations $\rho$, we relate the hybridized fraction $\theta(T)$ to the standard free energy via

$$
\ln K_{a}=\frac{\Delta G}{R T}=\frac{\Delta S}{R}-\frac{\Delta H}{R T}=\ln \frac{\theta(T) C^{\circ}}{[1-\theta(T)]^{2} \rho} .
$$

Thus, we obtain the enthalpy change and entropy change from the slope and intercept of a plot of $\ln K_{a}$ versus $1 / T$.

We confirm that the two-state model is accurate by performing the same experiment at different strand concentrations and inferring the enthalpy change and entropy change from an Arrhenius plot of $1 / T_{m}$ versus $\ln \rho$, where $T_{m}$ is defined as the temperature at which $\theta=0.5$. The values obtained from both methods agree to within statistical uncertainty.

To account for the possible influence of the poly-T spacers on the DNA thermodynamics, we use the full linker sequences and modified grafted sequences, which contain the last 12 nucleotides on the 3' ends. The enthalpy and entropy changes that we find are given in Supplemental Material Table II [22].

\section{Measuring the melting temperature}

We measure the melting temperature of our colloidal suspensions using optical microscopy. Samples are prepared by mixing particle species $A$, particle species $B$, and free single-stranded DNA solution in a $1: 1: 2$ ratio. The final solutions contain linker strands and competitor strands in 10-mM Tris, 1-mM EDTA, 500-mM NaCl, and have particle volume fractions of roughly $0.25 \%(\mathrm{v} / \mathrm{v})$ per species. Linker solutions and competitor solutions are prepared at various concentrations, which are measured directly using UV spectrophotometry (NanoDrop 2000c, Thermo Scientific). The final solution of free singlestranded DNA is created by combing these two solutions of known concentration.

Next, we prepare sample chambers using two cover slips (No. 1, VWR) that are bonded together and sealed with silicone vacuum grease (Dow Corning) and affixed with UV-curable optical adhesive (Norland 63) or quick-dry nail polish (big kwik dry top coat, Sally Hansen). The cover slips are plasma cleaned for approximately $1 \mathrm{~min}$ before the chamber is assembled in order to prevent nonspecific binding of the DNA-grafted particles and the chamber walls. Finished sample chambers are roughly $1.7 \mu \mathrm{L}$ in volume and about $70 \mu \mathrm{m}$ in height.

We prepare a separate microscope chamber for each unique combination of linker length, linker concentration, and particle grafting density. Once the chamber is assembled, we anneal the sample in an oven (Shell Lab, 1330FM Horizontal Airflow Oven) at $70^{\circ} \mathrm{C}$ for $30 \mathrm{~min}$ and then at $45^{\circ} \mathrm{C}$ for another $30 \mathrm{~min}$. The samples are then removed from the oven and allowed to sit at room temperature for at least $1 \mathrm{~h}$.

We image our samples on an inverted optical microscope (Nikon Eclipse TE2000-E) using a $60 \times$ oil-immersion objective (VC, Nikon). We control the sample temperature using two heating elements: (1) We heat our sample from below using an objective heater fitted with a thermosensitive resistor and driven by a low-noise temperature controller (BioScience Tools); (2) we also control the sample temperature from above using a Peltier heater (TE Technology) with a hole in its center, which is bonded to the sample chamber with optical gel (Cargille Optical Gel) and controlled via software (TC 720, TE Technologies). The Peltier is equipped with a water block.

We measure the melting temperature by slowly heating the sample while imaging it simultaneously using a digital camera. The temperature is incremented by $1{ }^{\circ} \mathrm{C}$, the sample is equilibrated for 5-10 min, and then digital images are acquired. The melting temperature is determined from this series of images by determining the lowest temperature at which approximately $50 \%$ of all particles are unbound. The singlet fraction is determined by visual inspection. Since the melting transition is abrupt (roughly $1{ }^{\circ} \mathrm{C}$ wide), we estimate an uncertainty of roughly $1{ }^{\circ} \mathrm{C}$ associated with our measurements of the melting temperature $T_{m}$. Samples that are disaggregated even at room temperature and do not have a measurable melting temperature are indicated by $x$ 's in the figures in the main text.

[1] W. B. Rogers, W. M. Shih, and V. N. Manoharan, Using DNA to Program the Self-Assembly of Colloidal Nanoparticles and Microparticles, Nat. Rev. Mater. 1, 16008 (2016).

[2] L. Di Michele and E. Eiser, Developments in Understanding and Controlling Self-Assembly of DNA-Functionalized Colloids, Phys. Chem. Chem. Phys. 15, 3115 (2013).

[3] M. R. Jones, N. C. Seeman, and C. A. Mirkin, Programmable Materials and the Nature of the DNA Bond, Science 347, 1260901 (2015).

[4] R. J. Macfarlane, B. Lee, M. R. Jones, N. Harris, G. C. Schatz, and C. A. Mirkin, Nanoparticle Superlattice Engineering with DNA, Science 334, 204 (2011).

[5] B. Srinivasan, T. Vo, Y. Zhang, O. Gang, S. Kumar, and V. Venkatasubramanian, Designing DNA-Grafted Particles that Self-Assemble into Desired Crystalline Structures Using the Genetic Algorithm, Proc. Natl. Acad. Sci. U.S.A. 110, 18431 (2013).

[6] R. J. Macfarlane, R. V. Thaner, K. A. Brown, J. Zhang, B. Lee, S. T. Nguyen, and C. A. Mirkin, Importance of the DNA Bond in Programmable Nanoparticle Crystallization, Proc. Natl. Acad. Sci. U.S.A. 111, 14995 (2014).

[7] Y. Wang, Y. Wang, X. Zheng, É. Ducrot, J. S. Yodh, M. Weck, and D. J. Pine, Crystallization of DNA-Coated Colloids, Nat. Commun. 6, 7253 (2015). 
[8] Y. Wang, I. C. Jenkins, J. T. McGinley, T. Sinno, and J. C. Crocker, Colloidal Crystals with Diamond Symmetry at Optical Lengthscales, Nat. Commun. 8, 14173 (2017).

[9] S. Hormoz and M. P. Brenner, Design Principles for SelfAssembly with Short-Range Interactions, Proc. Natl. Acad. Sci. U.S.A. 108, 5193 (2011).

[10] Z. Zeravcic, V. N. Manoharan, and M. P. Brenner, Size Limits of Self-Assembled Colloidal Structures Made Using Specific Interactions, Proc. Natl. Acad. Sci. U.S.A. 111, 15918 (2014).

[11] K.-T. Wu, L. Feng, R. Sha, R. Dreyfus, A. Y. Grosberg, N. C. Seeman, and P. M. Chaikin, Polygamous Particles, Proc. Natl. Acad. Sci. U.S.A. 109, 18731 (2012).

[12] P. L. Biancaniello, A. J. Kim, and J. C. Crocker, Colloidal Interactions and Self-Assembly Using DNA Hybridization, Phys. Rev. Lett. 94, 058302 (2005).

[13] R. Dreyfus, M. E. Leunissen, R. Sha, A. V. Tkachenko, N. C. Seeman, D. J. Pine, and P. M. Chaikin, Simple Quantitative Model for the Reversible Association of DNA Coated Colloids, Phys. Rev. Lett. 102, 048301 (2009).

[14] W. B. Rogers and J. C. Crocker, Direct Measurements of DNA-Mediated Colloidal Interactions and Their Quantitative Modeling, Proc. Natl. Acad. Sci. U.S.A. 108, 15687 (2011).

[15] J. SantaLucia, A Unified View of Polymer, Dumbbell, and Oligonucleotide DNA Nearest-Neighbor Thermodynamics, Proc. Natl. Acad. Sci. U.S.A. 95, 1460 (1998).

[16] A. J. Kim, V. N. Manoharan, and J. C. Crocker, SwellingBased Method for Preparing Stable, Functionalized Polymer Colloids, J. Am. Chem. Soc. 127, 1592 (2005).

[17] J. D. Halverson and A. V. Tkachenko, DNA-Programmed Mesoscopic Architecture, Phys. Rev. E 87, 062310 (2013).

[18] J. D. Halverson and A. V. Tkachenko, Communication: Programmable Self-Assembly of Thin-Shell Mesostructures, J. Chem. Phys. 147, 141103 (2017).

[19] S. Y. Park, A. K. R. Lytton-Jean, B. Lee, S. Weigand, G. C. Schatz, and C. A. Mirkin, DNA-Programmable Nanoparticle Crystallization, Nature (London) 451, 553 (2008).

[20] H. Xiong, D. van der Lelie, and O. Gang, Phase Behavior of Nanoparticles Assembled by DNA Linkers, Phys. Rev. Lett. 102, 015504 (2009).

[21] H. Xiong, D. van der Lelie, and O. Gang, DNA LinkerMediated Crystallization of Nanocolloids, J. Am. Chem. Soc. 130, 2442 (2008).

[22] See Supplemental Material at http://link.aps.org/ supplemental/10.1103/PhysRevX.9.041054 for the DNA base sequences and details about the theory.

[23] W. B. Rogers and V. N. Manoharan, Programming Colloidal Phase Transitions with DNA Strand Displacement, Science 347, 639 (2015).

[24] L. Di Michele, S. J. Bachmann, L. Parolini, and B. M. Mognetti, Communication: Free Energy of LigandReceptor Systems Forming Multimeric Complexes, J. Chem. Phys. 144, 161104 (2016).

[25] P. Varilly, S. Angioletti-Uberti, B. M. Mognetti, and D. Frenkel, A General Theory of DNA-Mediated and Other Valence-Limited Colloidal Interactions, J. Chem. Phys. 137, 094108 (2012).
[26] B. M. Mognetti, M.E. Leunissen, and D. Frenkel, Controlling the Temperature Sensitivity of DNA-Mediated Colloidal Interactions through Competing Linkages, Soft Matter 8, 2213 (2012).

[27] L. Parolini, J. Kotar, L. Di Michele, and B. M. Mognetti, Controlling Self-Assembly Kinetics of DNA-Functionalized Liposomes Using Toehold Exchange Mechanism, ACS Nano 10, 2392 (2016).

[28] R. P. Sear, The Stability Limit of the Fluid Phase of Polydisperse Sticky Spheres, Mol. Phys. 96, 1013 (1999).

[29] P. Charbonneau and D. Frenkel, Gas-Solid Coexistence of Adhesive Spheres, J. Chem. Phys. 126, 196101 (2007).

[30] W. B. Rogers and J. C. Crocker, Reply to Mognetti et al.: DNA Handshaking Interaction Data Are Well Described by Mean-Field and Molecular Models, Proc. Natl. Acad. Sci. U.S.A. 109, E380 (2012).

[31] T. C. Hales, A Proof of the Kepler Conjecture, Ann. Math. 162, 1065 (2005).

[32] M. Lee, J. W. Collins, D. M. Aubrecht, R. A. Sperling, L. Solomon, J.-W. Ha, G.-R. Yi, D. A. Weitz, and V. N. Manoharan, Synchronized Reinjection and Coalescence of Droplets in Microfluidics, Lab Chip 14, 509 (2014).

[33] C. L. Smith, S. A. Oñate, M.-J. Tsai, and B. W. O'Malley, Creb Binding Protein Acts Synergistically with Steroid Receptor Coactivator-1 to Enhance Steroid ReceptorDependent Transcription, Proc. Natl. Acad. Sci. U.S.A. 93, 8884 (1996).

[34] F. Zhang, M. W. A. Skoda, R. M. J. Jacobs, S. Zorn, R. A. Martin, C. M. Martin, G. F. Clark, S. Weggler, A. Hildebrandt, O. Kohlbacher et al., Reentrant Condensation of Proteins in Solution Induced by Multivalent Counterions, Phys. Rev. Lett. 101, 148101 (2008).

[35] T. T. Nguyen, I. Rouzina, and B. I. Shklovskii, Reentrant Condensation of DNA Induced by Multivalent Counterions, J. Chem. Phys. 112, 2562 (2000).

[36] R. Asor, O. Ben-nun-Shaul, A. Oppenheim, and U. Raviv, Crystallization, Reentrant Melting, and Resolubilization of Virus Nanoparticles, ACS Nano 11, 9814 (2017).

[37] D. B. Lukatsky and D. Frenkel, Phase Behavior and Selectivity of DNA-Linked Nanoparticle Assemblies, Phys. Rev. Lett. 92, 068302 (2004).

[38] D. B. Lukatsky and D. Frenkel, Surface and Bulk Dissolution Properties, and Selectivity of DNA-Linked Nanoparticle Assemblies, J. Chem. Phys. 122, 214904 (2005).

[39] D. Y. Zhang and G. Seelig, Dynamic DNA Nanotechnology Using Strand-Displacement Reactions, Nat. Chem. 3, 103 (2011).

[40] J. J. Hopfield, Kinetic Proofreading: A New Mechanism for Reducing Errors in Biosynthetic Processes Requiring High Specificity, Proc. Natl. Acad. Sci. U.S.A. 71, 4135 (1974).

[41] L. H. Hartwell, J. J. Hopfield, S. Leibler, and A. W. Murray, From Molecular to Modular Cell Biology, Nature (London) 402, C47 (1999).

[42] A. J. Kim, P. L. Biancaniello, and J. C. Crocker, Engineering DNA-Mediated Colloidal Crystallization, Langmuir 22, 1991 (2006).

[43] N. C. Seeman, De Novo Design of Aequences for Nucleic Acid Structural Engineering, J. Biomol. Struct. Dyn. 8, 573 (1990). 